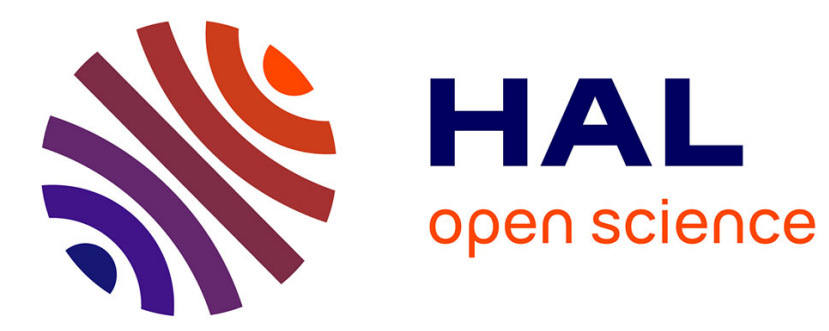

\title{
Chaotic behavior analysis based on corner bifurcations
}

\author{
Djamila Benmerzouk, Jean-Pierre Barbot
}

\section{To cite this version:}

Djamila Benmerzouk, Jean-Pierre Barbot. Chaotic behavior analysis based on corner bifurcations. Nonlinear Analysis: Hybrid Systems, 2009, 3 (4), pp.543-550. 10.1016/j.nahs.2009.04.005 . hal00772300

\section{HAL Id: hal-00772300 \\ https://hal.inria.fr/hal-00772300}

Submitted on 10 Jan 2013

HAL is a multi-disciplinary open access archive for the deposit and dissemination of scientific research documents, whether they are published or not. The documents may come from teaching and research institutions in France or abroad, or from public or private research centers.
L'archive ouverte pluridisciplinaire HAL, est destinée au dépôt et à la diffusion de documents scientifiques de niveau recherche, publiés ou non, émanant des établissements d'enseignement et de recherche français ou étrangers, des laboratoires publics ou privés. 


\title{
Chaotic behavior analysis based on corner bifurcations
}

\author{
D. Benmerzouk $k^{\mathrm{a}, 1}$, J.P. Barbot ${ }^{\mathrm{b}, *}$ \\ ${ }^{a}$ University of Tlemcen,Department of Mathematics,BP 119, 13000 Tlemcen, Algeria \\ ${ }^{b}$ Equipe Commande des Systèmes, ENSEA, Cergy-Pontoise Paris, France $\mathcal{G}^{3}$ \\ EPI-ALIEN-INRIA
}

\begin{abstract}
In this paper, a mathematical analysis in order to generate a chaotic behavior for piecewise smooth systems submitted to one of it's specific bifurcations, namely the corner one, is proposed. This study is based on period doubling method.
\end{abstract}

Key words: Bifurcation of limits cycles and periodic orbits (37G15), Existence of solutions (74H20), Chaotic behavior (74H65), Bifurcations connected with nontransversal intersection (37G25).

\section{Introduction}

Piecewise Smooth Systems (P.W.S) are dynamic systems given by two or more sets of differential equations such that those systems switch from one phase space (defined by smooth dynamics) to another one when some switching conditions are satisfied, those regions are separated by finitely smooth codimension one boundaries; In fact, discontinuities represent an important class of nonlinear dynamical systems, these systems exhibit complex behavior that cannot be explained only by using the bifurcation analysis associated to smooth dynamic systems [15], they can undergo all the bifurcations relied to smooth systems but they have also a specific type of bifurcations called border collision bifurcations, as grazing, sliding, chattering, corner... that occur when a fixed point collides with a borderline resulting in a change in the associated Jacobian matrix. Note that many papers deals with on piecewise smooth maps that are continuous across the borderlines [3, 4], however, many switching dynamic systems as DC-DC converters [5], thyristor controlled reactor circuits [23], digitally controlled systems [16] give rise to discontinuous maps. The bifurcation theory for one and two dimensional continuous piecewise maps has been developed in $[3,11,10,20,24]$. M. Feigin obtained some results on periodic orbits for continuous maps [13] and some authors extend the Feigin's approach to the case of one dimensional discontinuous maps [18]: the idea is to consider a piecewise linear approximation of the relied system in the neighborhood of the discontinuity plane in order to obtain general existence conditions of period-1, period

\footnotetext{
${ }^{*}$ Corresponding author

${ }^{1}$ This work was partially supported by the "Haut Conseil Franco-Algrien Universitaire et de Recherche"
} 
-2 fixed points before and after a border collision bifurcation. On the other hand, one of the most striking feature of piecewise smooth systems is that they exhibit sudden transitions from periodic attractors to chaos in the absence of any period doubling or other bifurcation cascades usually observed in smooth systems [19]. Hereafter treating the case of way to chaos: via grazing bifurcation in [6] and via the sliding one in [7], we consider the case of way to chaos via corner bifurcation: it is certainly the easier to understand graphically, but also one of the most tedious one to analyze. This is due to its specific topological structure (the discontinuity boundary is a corner -type formed by the intersection between two smooth codimension one surfaces at a nonzero angle) and to its very particular assumptions. The paper provides the foundations of a rational approach of transition to chaos by period doubling base on the relied Poincaré map introduced by di Bernardo and co-workers [10].

On the other hand, an important feature to consider is that there exist two types of chaotic attractors: the fragile chaos corresponding to the disappearance of the attractors or the coexistence of another attractors when some parameter is perturbed and the robust chaos corresponding to the absence of periodic windows that could destroy the chaotic behavior of the system for small perturbations of the bifurcation parameter. In many practical applications, it is of the most importance to maintain a robust chaotic comportment as in communications, electrical engineering [21, 22], so, an overview of some important issues concerning the robustness of chaos in smooth dynamic systems is given in $[1,2,12]$. The result obtained in [1] contradicts the conjecture that robust chaos cannot occur for smooth systems, here, the analysis concerns piecewise smooth systems submitted to corner bifurcations, our approach guaranties a robust chaotic behavior (in the meaning that for some small bifurcation parameter variations, the system behavior stays chaotic), the robustness analysis is different from those given in the previous results and is obtained directly from the proposed approach.

The paper is organized as follows: In section II, some recalls on the Poincaré map associated to the corner bifurcations are given and the problem statement is established; in section III, the periodic existence and uniqueness problem solution's analysis is presented based on the Implicit Functions theorem; a way to chaos by period doubling method is proposed in section IV. Finally, an example based on this approach with simulations results and comments are presented in section $\mathrm{V}$.

\section{Recalls and Problem statement}

Let us consider the following piecewise smooth system:

$$
\dot{x}=\left\{\begin{array}{c}
F_{1}(x) \text { if } H_{1}(x) \geq 0 \text { or } H_{2}(x) \geq 0 \\
F_{2}(x) \text { if } H_{1}(x)<0 \text { and } H_{2}(x)<0
\end{array}\right.
$$

where $x: I \longrightarrow D, D$ is an open bounded domain of $R^{n}$ with $n \geq 3, I$ is the time interval .

$$
F_{1}, F_{2}: C_{a b s}(I, D) \longrightarrow C^{k}(I, D), \quad k \geq 2 .
$$


where $C^{k}(I, D)$ is the set of $C^{k}$ functions defined on $I$ and having values in $R^{n}$, the norm for $C^{k}(I, D)$ is defined as follows:

$x \in C^{k}(I, D) \quad:\|x\|_{k}=\sup _{t \in I}\|x(t)\|_{n}+\sup _{t \in I}\|\dot{x}(t)\|_{n}+\ldots+\sup _{t \in I}\left\|x^{(k)}(t)\right\|_{n}$

$x^{(k)}($.$) is the k^{t h}$ derivative of $x(),.\|.\|_{n}$ is a norm defined on $R^{n}$ and $C_{a b s}(I, D)$ is the set of absolutely continuous functions defined on $I$ and having values in $D$ provided with the norm: $x \in C_{a b s}(I, D):\|x\|=\sup _{t \in I}\|x(t)\|_{n}$

According to [8], $\left(C^{k}(I, D),\|\|.\right)$ and $\left(C_{a b s}(I, D),\|\|.\right)$ are Banach spaces.

Now, let's consider for $i=1,2$ two $C^{1}$ applications: $H_{i}: D \longrightarrow R$ such that they define the sets:

$$
S_{1}^{0}=\left\{x(t) \in D: H_{1}(x(t))=0\right\} \text { and } S_{2}^{0}=\left\{x(t) \in D: H_{2}(x(t))=0\right\}
$$

It is assumed that $S_{1}^{0}$ and $S_{2}^{0}$ intersect along a corner (noted C) with a smooth codimension two surfaces, this generates a non-zero angle, now let's consider (without loss of generality) an initial point $\left(x_{0}, t_{0}\right)=(0,0)$ (after an eventual translation), such that $(0,0)$ is on the corner $\mathrm{C}$ i.e satisfies the following assumption:

Assumption:

$\mathrm{H}-1) \nabla H_{1}(0,0) \times \nabla H_{2}(0,0) \neq 0$.

Moreover, the following regions are not empty:

$D_{\text {int }}=\left\{x(t) \in D: H_{1}\left((x(t))<0\right.\right.$ and $H_{2}((x(t))<0\}$

$D_{\text {out }}=\left\{x(t) \in D: H_{1}(x(t)) \geq 0\right.$ or $\left.H_{2}(x(t)) \geq 0\right\}$

Both vector fields $F_{1}$ and $F_{2}$ are defined on both sides of $D_{\text {int }}$ and $D_{\text {out }}$ respectively.

Now, in order to define the corner-collision trajectory, two possibilities occur: external collision and internal one, where for the sake of clarity both collisions are given for systems of dimension 2 (see figure 1):

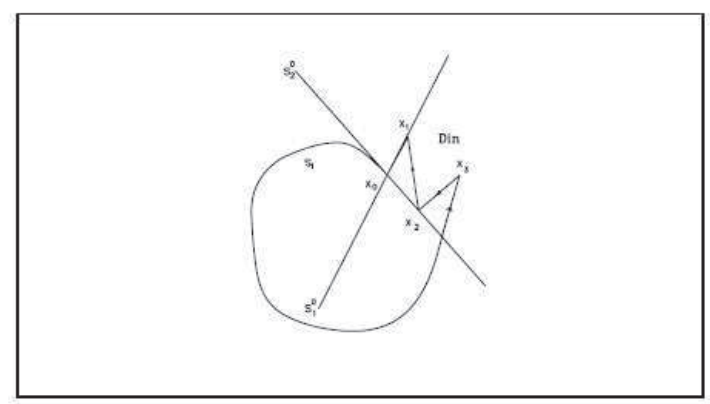

Figure 1: Poincaré map associated to corner collision

Remark 1. The internal corner becomes an external one by changing the corresponding obtuse angle to an acute one. So, the complete study of the internal case (for example) is sufficient.

So, in order to avoid sliding phenomena, one requires the following assumptions for each case: 


\section{Assumptions:}

For the external corner collision:

$\left.\mathrm{A}-1)<F_{i}(0,0), \nabla H_{1}(0,0)\right)><0$ and $<F_{i}(0,0), \nabla H_{2}(0,0)>>0, i=1,2$.

For the internal corner collision:

$\left.\mathrm{A}-2)<F_{i}(0,0), \nabla H_{1}(0,0)\right)>\geq 0$ or $\left\langle F_{i}(0,0), \nabla H_{2}(0,0)>\leq 0, i=1,2\right.$.

where $\langle.,$.$\rangle is a usual scalar product on R^{n}$.

As it was highlighted in the introduction, the system (1) characterizes the behavior of an increasing number of dynamic systems in applied sciences and engineering, those piercewise smooth systems can present a specific switching bifurcations particularly when depending on some parameter such as grazing, chattering, sliding,... So, in order to present a complete bifurcation analysis, one assumes that the system (1) depends smoothly on a parameter $\varepsilon$ defined in a neighborhood $V_{\varepsilon}$ of 0 such that at $\varepsilon=0$, there exists a periodic orbit $x(t):=p(t)$ that intersects with the corner $\mathrm{C}$ at the point $x^{*}=0=p(0)$ corresponding to $t^{*}$ (where $t^{*}$ is defined modulo the periodicity of (1)) and that there exists a neighborhood of $x$ noted $V_{x}$ such that there is no other points of collision near $x^{*}$, so by continuity, one can assume that this will be also true locally for a sufficiently small $\varepsilon$.

Consequently, the system (1) is rewrite as follows:

$$
\dot{x}=\left\{\begin{array}{c}
F_{1}(x, \varepsilon) \text { if } H_{1}(x) \geq 0 \text { or } H_{2}(x) \geq 0 \\
F_{2}(x, \varepsilon) \text { if } H_{1}(x)<0 \text { and } H_{2}(x)<0
\end{array}\right.
$$

Such that $p(t)$ is an $\mathrm{T}$ periodic orbit, thus for $(x, \varepsilon)$ in the neighborhood of $(0,0) \epsilon V_{x} \times V_{\varepsilon}$ and using the smooth dependence of the parameters on $V_{\varepsilon}$ and the lack of further grazing, sliding or corner collisions outside $V_{x}$ permits to define the time - $\mathrm{T}$ flow map noted $\Pi_{p e r}$ as follows:

$$
\Pi_{p e r}:(x, \varepsilon) \rightarrow \Pi_{p e r}(x, \varepsilon)=P x+Q \varepsilon+o(x, \varepsilon)
$$

Where $P$ is a $n \times n$ nonsingular matrix, $Q$ is a $n$ dimensional non null vector and $o(x, \varepsilon) \longrightarrow 0$ when $(x, \varepsilon) \longrightarrow(0,0)$.

Two different cases of bifurcations involving corner can occur:

Case 1, internal bifurcation: the construction of the corresponding Zerotime Discontinuity Map (noted ZDM) is done according to [10] and the corresponding Poincaré map takes the form:

$$
\Pi_{p e r}^{*}:(x, \varepsilon) \rightarrow \Pi_{p e r}^{*}(x, \varepsilon)=P^{*} x+Q^{*} \varepsilon+o(x, \varepsilon) .
$$

Where $P^{*}$ is a $(n-1) \times(n-1)$ nonsingular matrix, $Q^{*}$ is a $(n-1)$ dimensional non null vector. Thus, when the considering trajectory enters in $D_{i n}$ in the neighborhood of $\mathrm{C}$, one obtains the following Poincare normal form by the composition of $\Pi_{\text {per }}^{*}$ with the ZDM:

$$
P_{1}(x, \varepsilon)= \begin{cases}P x+Q \varepsilon+o(x, \varepsilon) & \text { if non-crossing } \\ P x+\left(F_{1}-F_{2}\right)<a_{2}, x>+Q \varepsilon+o(x, \varepsilon) & \text { if crossing }\end{cases}
$$

where:

$$
a_{2}=J_{2}-\frac{1}{2}<J_{2}, F_{1}>J_{1}, J_{i}=\frac{\nabla H_{i}}{<\nabla H_{i}, F_{i}>}, i=1,2
$$


and for the sake of compactness, $F_{1}, F_{2}$ and $\nabla H$ stand (and also for the next case) respectively for $F_{1}(0), F_{2}(0)$ and $\nabla H_{i}(0)$.

Case 2, external bifurcation: the local analysis permitting to define the Poincaré map is different in computations than the previous case but the principle is the same: the construction of the ZDM in done according to [10] and by composing with its flow map, the corresponding Poincaré normal form is given by:

$P_{2}(x, \varepsilon)= \begin{cases}P x+Q \varepsilon+o(x, \varepsilon) & \text { if non-crossing } \\ P x+\left(F_{1}-F_{2}\right)<a_{1}, x>+Q \varepsilon+o(x, \varepsilon) & \text { if crossing }\end{cases}$

where

$$
a_{1}=J_{1}-<J_{1}, F_{2}>J_{2}
$$

Note that for the sake of notation simplicity, the projection of $x$ on the Poincaré section and its corresponding neighborhood are also noted respectively by $x$ and $V_{x}, P^{*}$ is noted $P$ and $Q^{*}$ is noted $Q$.

\section{Problem analysis}

In this section, local existence an uniqueness of periodic solutions for problem (2) are investigated for the two cases; Knowing that searching a periodic orbit of (2) is equivalent to determine a fixed point of its Poincaré map $P_{i}(., \varepsilon)$, then the problem becomes to resolve for each case $i=1,2$ the following equation:

$$
P_{i}(x, \varepsilon)=x, \text { where }(x, \varepsilon) \epsilon V_{x} \times V_{\varepsilon}
$$

So, both possibilities are considered:

\section{First possibility: there is no crossing:}

Thus, one only considers the first equation of the system (2) and the problem becomes to analyze the same equation for the two cases given by:

$$
\gamma(x, \varepsilon)=P x+Q \varepsilon-x+o(x, \varepsilon)=0, \text { where }(x, \varepsilon) \epsilon V_{x} \times V_{\varepsilon}
$$

Now, in order to use the Implicit Functions theorem, the following assumption is necessary:

Assumption:

$\mathrm{H}-2)\left(P-I_{d}\right)$ is a nonsingular matrix, where $I_{d}$ is the $(n-1) \times(n-1)$ identity matrix.

And it comes:

Lemma 1. Under assumptions $H$-1), $H$-2) and $\left.A_{i}\right)$ associated to each case $i=1,2$, there exist a neighborhood $v_{\varepsilon=0} \subset v_{\varepsilon}$ in $R$, a neighborhood $\nu_{x=0}$ on the Poincaré section's (defined in $R^{n-1}$ ) and an unique application $x^{*}: v_{\varepsilon=0} \longrightarrow$ $\nu_{x=0}$ solution of $\gamma\left(x^{*}(\varepsilon), \varepsilon\right)=0$ such that $x^{*}(0)=0$, furthermore, the periodic solution $x^{*}$ of (2) depends continuously on $\varepsilon$. 


\section{Second possibility: a crossing occur:}

Thus the problem of finding a periodic solution of problem (2) when crossing occurs is equivalent to analyze for each case $i=1,2$, the following corresponding equations:

for case 1, (internal collision bifurcation):

$$
\beta_{1}(x, \varepsilon)=P x+\left(F_{1}-F_{2}\right)<a_{2}, x>+Q \varepsilon+o(x, \varepsilon)-x=0
$$

where $a_{2}$ is given by $(4)$ and $(x, \varepsilon) \epsilon V_{x} \times V_{\varepsilon}$.

for case 2, (external collision-bifurcation):

$$
\beta_{2}(x, \varepsilon)=P x+\left(F_{1}-F_{2}\right)<a_{1}, x>+Q \varepsilon+o(x, \varepsilon)-x=0
$$

where $a_{1}$ is given by $(6)$ and $(x, \varepsilon) \epsilon V_{x} \times V_{\varepsilon}$.

So, as for the first case (of non-crossing) and in order to use the Implicit functions theorem, the following assumptions are needed relatively to each case $i=1,2$ :

Assumptions:

B-i) $B_{i}=\left(P-I_{d}+\left(F_{1}-F_{2}\right) a_{i}^{T}\right)$ is a nonsingular matrix.

Thus, one obtains:

Lemma 2. Under assumptions $H-1$ ) and $A$-i), B-i) specific to each case $i=$ 1,2 , there exist a neighborhood $v_{\varepsilon=0}^{i} \subset v_{\varepsilon}$ in $R$, a neighborhood $\nu_{x=0}^{i}$ on the Poincaré section's (defined in $R^{n-1}$ ) and an unique application $x_{i}^{* *}: v_{\varepsilon=0}^{i} \longrightarrow$ $\nu_{x=0}^{i}$ solution of $\beta_{i}\left(x_{i}^{* *}(\varepsilon), \varepsilon\right)=0$ such that $x_{i}^{* *}(0)=0$, furthermore, the periodic solution $x^{* *}$ of (2) depends continuously on $\varepsilon$.

Now, after analyzing the problem of existence and uniqueness of solutions for piecewise smooth systems submitted to corner bifurcations, the aim of the next section is to present a mathematical approach in order to generate chaotic behavior for this type of systems.

\section{Way to Chaos}

First, recall that there exist three types of dynamic systems:

1-Stochastic systems that evolve with hazard in the space such that they have no representing equations and any exact prevision of their comportment is possible.

2-The deterministic systems that are represented by known mathematical equations permitting to predict exactly the evolution of these systems at any fixed time.

3-The chaotic systems that are also deterministic but have an infinitely more complex behavior characterized by two features:

3-1) A great sensitivity to initial conditions (measured by the biggest Lyapounov exponent).

3-2) The complex structure of its attractor (called strange attractor and measured by the topological entropy [14]). 
The aim of this work is to present a mathematical analysis permitting to generate a deterministic chaos for piecewise smooth systems given by the form (2) and that are submitted to those specific bifurcations namely the corner ones, so according to [14] or [25], one way to generate chaotic behavior for one dimensional discrete systems is those based on period doubling. Nevertheless, simulations show that it is also possible to generate chaos for piecewise smooth systems of dimension greater than 1 . In this section, we propose a mathematical analysis for p.w.s systems of dimension 3 and by choosing a Poincaré map orthogonal to the surface, the considered system will be defined in dimension 1, this enables us to invoke the famous result (see for example [25]): "Period three implies chaos". More precisely, the problem becomes to determine for each map $\beta_{i}, i=1,2$, three distinct points noted respectively $x_{i}, y_{i}$ and $z_{i}$ such that: $\beta_{i}\left(x_{i}, \varepsilon\right)=y_{i}, \beta_{i}\left(y_{i}, \varepsilon\right)=z_{i}$ and $\beta_{i}\left(z_{i}, \varepsilon\right)=x_{i}$; this well be done naturally in three steeps as follows:

First step: analyze of the equation:

$$
\beta_{i}\left(x_{i}, \varepsilon\right)=y_{i}=x_{i}+\eta_{i}, \quad\left(x_{i}, \varepsilon\right) \epsilon V_{x_{i}} \times V_{\varepsilon}
$$

where for a sake of simplicity, $\eta_{i}$ stands for a vector defined in $R^{n-1}$, having only one component (namely the $j$ one, where $j$ is some fixed value between 1 and $n-1$ ) equal to some fixed value (noted also $\eta_{i}$ ) and the others components are nulls.

Thus, the equation (11) is equivalent to:

$$
\Psi_{i}\left(x_{i}, \varepsilon, \eta_{i}\right):=\beta_{i}\left(x_{i}, \varepsilon\right)-x_{i}-\eta_{i}=0, \quad\left(x_{i}, \varepsilon\right) \epsilon V_{x_{i}} \times V_{\varepsilon}
$$

And by considering the following assumptions for each case $i=1,2$ :

Assumptions:

C-i) $C_{i}=\left(P-2 I_{d}+\left(F_{1}-F_{2}\right) a_{i}^{T}\right)$ is a nonsingular matrix.

One obtains (with the same arguments than the previous results):

Lemma 3. Under assumptions $H-1), A$-i), and $C$-i) specific to each case $i=$ 1,2 , there exist a neighborhood $\vartheta_{\varepsilon=0}^{i} \subset v_{\varepsilon=0}^{i}$ in $R$, a neighborhood $v_{\eta_{i}}$ in $R$, a neighborhood $v_{x_{i}=0}$ on the Poincaré section's (defined in $R^{n-1}$ ) and an unique application $x_{i}^{* * *}: \vartheta_{\varepsilon=0}^{i} \times v_{\eta_{i=0}} \longrightarrow v_{x_{i}=0}$ solution of $\Psi_{i}\left(x_{i}^{* * *}\left(\varepsilon, \eta_{i}\right), \varepsilon, \eta_{i}\right)=0$ such that $x_{i}^{* * *}(0,0)=0$, furthermore, $x_{i}^{* * *}$ depends continuously on $\varepsilon$ and $\eta_{i}$.

Second step: analyze of the equation:

$$
\beta_{i}\left(\beta_{i}\left(x_{i}, \varepsilon\right), \varepsilon\right)=z_{i}=y_{i}+\mu_{i}=x_{i}^{* * *}\left(\varepsilon, \eta_{i}\right)+\eta_{i}+\mu_{i}
$$

where $\mu_{i}$ stands for a vector defined on $R^{n-1}$, having only one component equal to some fixed value(noted also $\mu_{i}$ ) and the others are nulls, thus, the equation (13) is equivalent to:

$$
\Gamma_{i}\left(\varepsilon, \eta_{i}, \mu_{i}\right):=\beta\left(x_{i}^{* * *}\left(\varepsilon, \eta_{i}\right)+\eta_{i}, \varepsilon\right)-x_{i}^{* * *}\left(\varepsilon, \eta_{i}\right)-\eta_{i}-\mu_{i}=0
$$

and in order to continue the process, the following assumptions (according to each case) are necessary: 


\section{Assumptions:}

D-i) $D_{i}=\left(B_{i} \tilde{C}_{j}+I_{d}^{0}\right)-\tilde{C}_{j}-I_{d}^{0}$ is a vector such that it's $j$ component (noted $d_{j}$ ) is non null.

where $\widetilde{C}_{j}$ is the $j$ column of the inverse matrix of $C_{i}$ and $I_{d}^{0}$ is the vector that only the $j$ component is equal to 1 and the other components are null.

And one obtains:

Lemma 4. Under assumptions $H-1), A-i), C$-i) and D-i) specific to each case $i=1,2$, there exist a neighborhood $v_{\varepsilon=0}^{i} \subset \vartheta_{\varepsilon=0}^{i}$, a neighborhood $\nu_{\eta_{i=0}} \subset v_{\eta_{i=0}}$, a neighborhood $\nu_{\mu_{i}=0}$ in $R$ and an unique application $\eta_{i}^{*}: v_{\varepsilon=0}^{i} \times \nu_{\mu_{i}=0} \longrightarrow$ $\nu_{\eta_{i=0}}$ solution of $\Gamma_{i}\left(\varepsilon, \eta_{i}^{*}\left(\varepsilon, \mu_{i}\right), \mu_{i}\right)=0$ such that $\eta_{i}^{*}(0,0)=0$, furthermore, $\eta_{i}^{*}$ depends continuously on $\varepsilon$ and $\mu_{i}$.

Third step: analyze of the equation:

$$
\beta_{i}\left(\beta_{i}\left(\beta_{i}\left(x_{i}, \varepsilon\right), \varepsilon\right), \varepsilon\right)=x_{i}
$$

So, taking account the previous results obtained in first and second steps, thus analyzing equation (15) is equivalent to analyze the following one:

$$
\begin{aligned}
\Pi_{i}\left(\varepsilon, \mu_{i}\right) & \left.:=\beta_{i}\left(x^{* * *}\left(\varepsilon, \eta_{i}^{*}\left(\varepsilon, \mu_{i}\right)\right)+\eta_{i}^{*}\left(\varepsilon, \mu_{i}\right)+\mu_{i}\right), \varepsilon\right) \\
& -x_{i}^{* * *}\left(\varepsilon, \eta_{i}^{*}\left(\varepsilon, \mu_{i}\right)\right)-\eta_{i}^{*}\left(\varepsilon, \mu_{i}\right)-\mu_{i}=0
\end{aligned}
$$

and in order to use the Implicit Functions theorem, the following assumptions are required for each case $i=1,2$ :

\section{Assumptions:}

E-i) $E_{i}=B_{i}\left(\tilde{C}_{j} \alpha+\alpha I_{d}^{0}+I_{d}^{0}\right)-\tilde{C}_{j} \alpha$ is a non null scalar, where $\alpha=-\frac{1}{d_{j}}$, And it comes:

Lemma 5. Under assumptions $H-1), A-i), C-i), D-i)$ and E-i) specific to each case $i=1,2$, there exist a neighborhood $\omega_{\varepsilon=0}^{i} \subset v_{\varepsilon=0}^{i}$, a neighborhood $\theta_{\mu_{i=0}}$ in $\nu_{\eta_{i=0}}$ and an unique application $\mu_{i}^{*}: \omega_{\varepsilon=0}^{i} \longrightarrow \theta_{\mu_{i=0}}$ solution of $\Pi_{i}\left(\varepsilon, \mu_{i}^{*}(\varepsilon)\right)=$ 0 such that $\mu_{i}^{*}(0)=0$, furthermore, $\mu_{i}^{*}$ depends continuously on $\varepsilon$.

The next corollary sums up the results obtained after the three steps necessary to generate period-doubling:

Corollary 6. Under assumptions H-1), A-i), C-i), D-i) and E-i) specific to each case $i=1,2$, the system (2) admits a chaotic behavior.

Comparing this result to the previous ones given in $[6,7]$, that correspond to the grazing and the sliding bifurcations, highlights the fact that corner bifurcations need less restrictive assumptions, consequently, corner bifurcations seem better choice for many applications as chaotic emitter design, DC-DC converters,...

Note also that this approach can also be extended to piecewise smooth systems of dimension $n$ greater than 3 and such that the dimension of the sliding surface (noted $d$ ) satisfies $n-d=2$. 


\section{An illustrative example}

Let us consider the following piercewise smooth system:

$$
\left\{\begin{array}{l}
\dot{x}_{1}=f_{11}\left(x_{1}, x_{2}, x_{3}\right) \\
\left.\dot{x}_{2}=f_{12}\left(x_{1}, x_{2}, x_{3}\right)\right) \\
\dot{x}_{3}=f_{13}\left(x_{1}, x_{2}, x_{3}\right)
\end{array} \quad \text { if } x \in D_{\text {int }}\right.
$$

and

$$
\left\{\begin{array}{l}
\dot{x}_{1}=f_{21}\left(x_{1}, x_{2}, x_{3}\right) \\
\dot{x}_{2}=f_{22}\left(x_{1}, x_{2}, x_{3}\right) \\
\dot{x}_{3}=f_{23}\left(x_{1}, x_{2}, x_{3}\right)
\end{array} \quad \text { if } x \in D_{\text {out }}\right.
$$

where:

$$
\begin{aligned}
& f_{11}\left(x_{1}, x_{2}, x_{3}\right)=x_{2}-\left(x_{1}^{2}+x_{2}^{2}-\left(1+10 \varepsilon\left(\sin \left(777 x_{3}\right)+1\right)^{2}\right)\right) x_{1} \\
& f_{12}\left(x_{1}, x_{2}, x_{3}\right)=-x_{1-}\left(x_{1}^{2}+x_{2}^{2}-\left(1+10 \varepsilon\left(\sin \left(777 x_{3}\right)+1\right)^{2}\right)\right) x_{2} \\
& f_{13}\left(x_{1}, x_{2}, x_{3}\right)=\varepsilon\left(x_{3}-\cos \left(330 x_{2}\right)-\left(x_{3}-\sin x_{2}\right)^{3}\right)
\end{aligned}
$$

and

$$
\begin{aligned}
& f_{21}\left(x_{1}, x_{2}, x_{3}\right)=\frac{x_{2}}{\pi}-100 x_{3}^{2}\left(\left(x_{1}+2\right)^{2}+x_{2}^{2}-\left(3+10 x_{3}\right)^{2}\right) x_{1} \\
& f_{22}\left(x_{1}, x_{2}, x_{3}\right)=-\left(\frac{x_{1}+2}{\pi}\right)-100 x_{3}^{2}\left(\left(x_{1}+2\right)^{2}+x_{2}^{2}-\left(3+10 x_{3}\right)^{2}\right) x_{2} \\
& f_{23}\left(x_{1}, x_{2}, x_{3}\right)=10 \pi \varepsilon\left(x_{3}+\sin \left(500 x_{1} x_{3}\right)\right)-\varepsilon x_{3}^{3}
\end{aligned}
$$

The functions that define borders $S_{1}^{0}, S_{2}^{0}$ and domains $\mathrm{D}_{\text {in }}$ and $\mathrm{D}_{\text {out }}$ are: $H_{1}\left(x_{1}, x_{2}, x_{3}\right)=-x_{1}+1$ and $H_{2}\left(x_{1}, x_{2}, x_{3}\right)=-x_{2}$ such that:

$S_{1}^{0}=\left\{\left(x_{1}, x_{2}, x_{3}\right) \in \mathbb{R}^{3}: H_{1}\left(x_{1}, x_{2}, x_{3}\right)=0\right\}$

$S_{2}^{0}=\left\{\left(x_{1}, x_{2}, x_{3}\right) \in \mathbb{R}^{3}: H_{2}\left(x_{1}, x_{2}, x_{3}\right)=0\right\}$

$\mathrm{D}_{\text {int }}=\left\{\left(x_{1}, x_{2}, x_{3}\right) \in \mathbb{R}^{3}: H_{1}\left(x_{1}, x_{2}, x_{3}\right)<0\right.$ and $\left.H_{2}\left(x_{1}, x_{2}, x_{3}\right)<0\right\}$

$\mathrm{D}_{\text {out }}=\left\{\left(x_{1}, x_{2}, x_{3}\right) \in \mathbb{R}^{3}: H_{1}\left(x_{1}, x_{2}, x_{3}\right) \geq 0\right.$ or $\left.H_{2}\left(x_{1}, x_{2}, x_{3}\right) \geq 0\right\}$

The conditions for internal collision are satisfied and computations give us the corresponding Poincaré map:

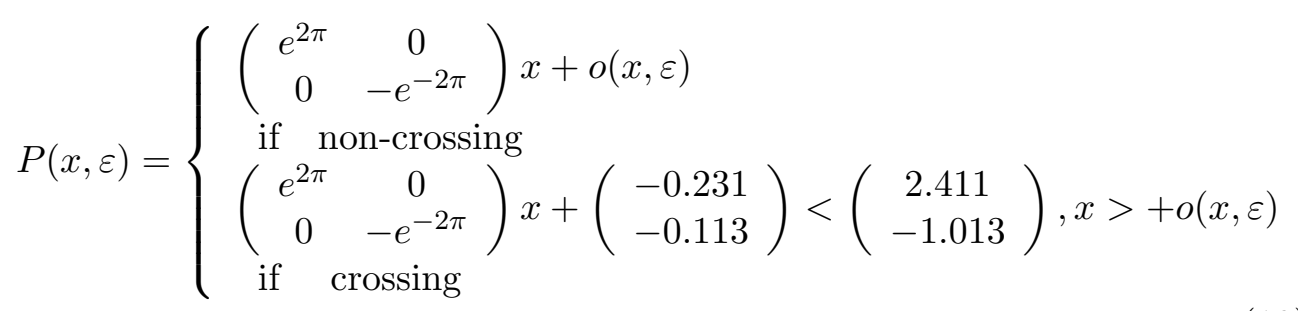

where $x=\left(x_{1}, x_{2}\right)^{T}$ and $o(x, \varepsilon) \longrightarrow 0$ when $(x, \varepsilon) \longrightarrow(0,0)$. 
For all simulations, the system is initialized in $x_{1}(0)=1.1, x_{2}(0)=0$, $x_{3}(0)=0$.

Moreover, in order to highlight the proposed way to chaos different values of $\varepsilon$ in zero's vicinity are considered:

- For $\epsilon=0$, the system (17-18) generates an attractive limit cycle, see figure 2 .

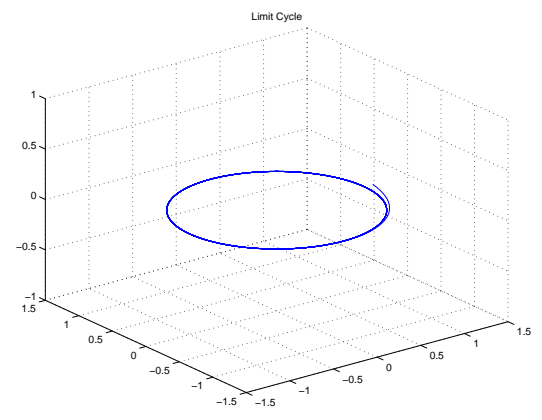

Figure 2: Limit Cycle for $\epsilon=0$

- $\underline{\text { For } \epsilon=0.001}$, a period doubling appears see figure 3 .

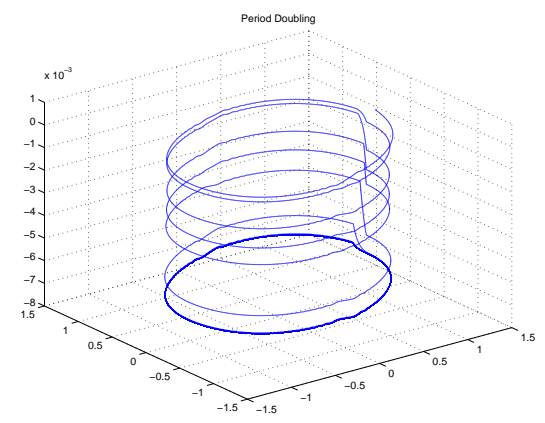

Figure 3: Period Doubling for $\epsilon=0.001$

- $\underline{\text { For } \epsilon=0.1}$, a chaotic behavior occurs see figure 4 .

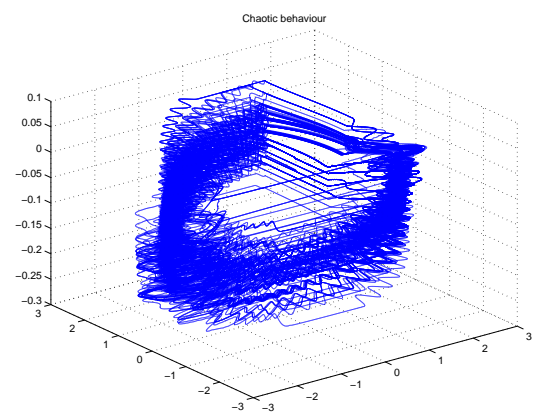

Figure 4: Chaotic Behavior $\epsilon=0.1$

These simulation results are in accord with the well founded of the proposed analysis (at least) for the considered system (17) and (18). 


\section{Conclusion}

In this paper, we have proposed a mathematical analysis for generating chaos with respect to piecewise smooth systems submitted to corner bifurcations. This approach shows that route to chaos is available not only for some fixed value of the bifurcation parameter but also for any value of this parameter in a neighborhood of this value. Consequently, it is possible to generate robust chaos for piecewise smooth systems submitted to corner bifurcations.

Obviously, this study is pertinent for bounded continuous subsystems at least of dimension 3. This work is based on the conjecture that "period three implies chaos" is also valid for discrete systems of dimension two and upper. The well-founded of this approach is highlighted by simulations results. Their are many possible extensions to this work, for example generating chaos for piecewise smooth systems with at least three different subsystems, more than one corner domains, piecewise smooth systems submitted simultaneously to different types of border bifurcations (for example grazing, sliding, corner), piecewise smooth systems having more than one bifurcation parameter,...

On the other hand, our analysis for corner (and also for sliding and grazing bifurcations) may be a useful tool for study the way to chaos in a general hybrid system composed of discrete events subsystems and piecewise smooth subsystems. At our point of view, the only difference in this case with our proposed approach is that each Poincaré map is function of the discrete event state. For such class of hybrid system, application domains are huge and include: many piecewise smooth systems driven by computers, many biological systems,...

\section{References}

[1] M.Andrecut and M.K.Ali,"Example of robust chaos in smooth map", Europhys.Lett.B", 2001, vol 54, n 3, pp300-305.

[2] M. Andrecut and M.K. Ali, "Robust chaos in smooth unimodal maps",Phys.Rev , E 64, 025203 (R)-3, 2001.

[3] S. Banergee and C. Grebogi, "Border collision bifurcations in 2-dimensional piecewise smooth maps "Phys.Rev.E, 59(4),4052-4061, 1999.

[4] S. Banergee, M.S.Karthik,G.H.Yuan and J.A.Yorke,"Bifurcations in one dimensional piecewise smooth maps", Theory and Applications in switching circuits.IEEE.Trans.Circuits.Syst.I47(3), 389-394,2000.

[5] S. Banergee, S. Parui and A. Gupta "Dynamic effects of missed switching in current-mode controlled DC-DC converters", IEEE Trans.Circuits Syst.II 51512, 646-654, 2004.

[6] D. Benmerzouk and J.P. Barbot, "Grazing analysis for synchronization of chaotic hybrid systems", Nolcos 2007, vol 1, 2007.

[7] D. Benmerzouk and J.P. Barbot, "Chaotic behavior Analysis based on sliding bifurcation", submitted for publication. 
[8] H. Bresis," Analyze fonctionnelle, Théorie et Applications", Dunod, Paris, 1999.

[9] M. di Bernardo, M.I. Feigin, S.J. Hogan and M.E. Homer, "Local Analysis of C-bifurcations in n-dimensional piecewise smooth dynamic systems", Chaos, Solitons,Fract.10(11), 1881-1908, 1999.

[10] M. di Bernardo, C.J. Budd and A.R. Champneys, "Corner-collision implies border-collision bifurcation" Physica D, 154:171-194, 2001.

[11] M. di Bernardo, C.J. Budd, A.R. Champneys, "Unified framework for the analysis of grazing and border-collisions in piecewise smooth systems", Physical Review Letters, 86 (12),2554-2556, 2001.

[12] Z. Elhadj and J.C.S prott," On the robustness of chaos in dynamic systems:Theories and Applications"; Front, Phys.China, 3(2):195-204, 2008.

[13] M.I.Feigin, "On the structure of C-bifurcation boundaries of piecewise continuous systems", Prikl.Mat.Mekh.42, 820-829 (1978), in Russian.

[14] P. Glendinning, "Stability, instability and chaos: An introduction to the theory of nonlinear differential equations", Cambridge University, Press, Cambridge.

[15] M. Golubitsky and D.G.Schaffer, "Singularities and groups in bifurcation theory";Vol.I,Applied Mathematical Sciences 51, Springer-Verlag, NewYork, 1985.

[16] G. Haller and G.Stepan,"Micro-chaos in digital control",J.Nonlinear Sci,6,415-448, 1996.

[17] H.K. Khalil, "Nonlinear systems", Prentice Hall, Second Edition, 1996.

[18] L.E. Kollar,G. Stepan and J. Turi, "Dynamics of piecewise linear discontinuous maps";Int.J.Bifurc.Chaos,14, 2341-2351, 2004.

[19] H.E. Nuss, E. Ott and J.A. Yorke, "Border collision bifurcations :An explanation for observed bifurcation phenomena", Phys.Rev.E.49,1073-1076, 1996.

[20] L.E. Nuss and J.A. Yorke, "Border-collision bifurcations for piecewise smooth one dimensional map", International Journal of bifurcation and chaos, Vol5, pp 189-207, 1995.

[21] J.M. Ottino, "The kinematics of mixing:Stretching, chaos and transport", Cambridge:Cambridge University Press,1989.

[22] J.M.Ottino,F.J.Muzzion,M.Tjahjadi,J.G.Franjione,S.C.Jana and H.A.Kurch ,Science, 257:754, 1992.

[23] R. Rajaraman ,I. Dobson and S. Jalali, "Nonlinear dynamics and switching time bifurcations of a thyristor controlled reactor circuit",IEEE Trans. Circuit Systems.I 43 (12),1001-1006 (1996). 
[24] S.Jaguste, K.M. Moudgalya, "A class of discontinuous dynamic systems, an industrial slurry high density polyethylene reactor", Chemical Engineering Science, 56,3611-3621, 2001.

[25] S. Wiggins, "Introduction to applied nonlinear dynamic systems and Chaos", Texts in Applied Mathematics 2, Springer-Verlag, 1990. 